COMMUNICATIONS IN

ANALYSIS AND GEOMETRY

Volume 13, Number 4, 741-768, 2005

\title{
Finite propagation speed for solutions of the parabolic $p$-Laplace equation on manifolds
}

\author{
S.A.J. (FIEKE) DEKKERS ${ }^{1}$
}

\begin{abstract}
We consider a class of degenerate parabolic equations containing the parabolic $p$-Laplace equation, on Riemannian manifolds. We prove that, on arbitrary manifolds, bounded solutions of such equations have finite propagation speed, and show that the rate of propagation can be estimated in terms of bounds on the Ricci curvature. The main technical tool in the proof is a new mean value type inequality for bounded solutions.
\end{abstract}

\section{Introduction.}

We consider solutions on an $n$-dimensional Riemannian manifold $M$ of a class of degenerate parabolic equations modelled on the parabolic $p$-Laplace equation

$$
u_{t}=\triangle_{p} u,
$$

where

$$
\triangle_{p} u=\operatorname{div}\left(|\nabla u|^{p-2} \nabla u\right),
$$

and $u=u(x, t), x \in M, t \geq 0$.

We assume $p>2$. This condition on $p$ implies that the equation degenerates at at points where $\nabla u=0$.

Not only is Equation (1.1) one of the simplest possible generalisations of the heat equation, it also has applications in fluid dynamics, where it describes the non-stationary flow in a porous medium of a non-Newtonian fluid, see [13] and references therein. Additionally, Equation (1.1) describes the propagation of heat after the explosion of a hydrogen bomb in the atmosphere [15]. This has motivated much of the research concerning equations such as (1.1). An account of the theory in $\mathbb{R}^{n}$ is given in [8], where the degenerate case $p>2$ is considered as well as the singular case $1<p<2$.

\footnotetext{
${ }^{1}$ Partially supported by Stichting Fonds Doctor Catharine van Tussenbroek
} 
See also, for example, $[7,9,13,14,15]$ and references therein. In contrast, there appears to be almost no literature concerning equations such as (1.1) on manifolds. Our study of these equations on manifolds is motivated by the fact that solutions of the heat equation on a Riemannian manifold are sensitive to the geometry of the manifold, and it seems natural to ask if this is true for solutions of the non-linear generalisation (1.1) of the heat equation, too.

In this paper, we consider one aspect of the behaviour of solutions of equations modelled on (1.1): their propagation speed. The behaviour of solutions of the degenerate Equation (1.1) with $p>2$ on $\mathbb{R}^{n}$ differs markedly from that of solutions of the heat equation, equation (1.1) with $p=2$, since unlike solutions of the heat equation, bounded solutions of $(1.1)$ on $\mathbb{R}^{n}$ have finite propagation speed. By this, we mean that the support of $u(\cdot, t)$ is contained in an $r$-neighbourhood of the support of the initial data, where $r=r(t)<\infty$.

On $\mathbb{R}^{n}$, there are several ways to prove this. One proof, see [8], involves a comparison with a known explicit solution, the so-called Barenblatt solution $[3,18]$. A similar approach is followed in $[1,4,7]$. Another proof (see $[9,14])$ uses the Sobolev inequality in $\mathbb{R}^{n}$. If we change the setting to a Riemannian manifold, where no analogue of the Barenblatt solution is available and Sobolev inequalities analogous to that in $\mathbb{R}^{n}$ do not in general hold, neither of these approaches works, and the question arises if finite propagation speed is a particular property of solutions of (1.1) on $\mathbb{R}^{n}$, or a generic property of solutions on Riemannian manifolds. We prove that the latter is the case, and that for solutions of the parabolic $p$-Laplace equation (which will be defined in Section 2) the following theorem holds (cf. Theorem 4.1):

Theorem 1.1. Assume that $M$ is a non-compact, complete, Riemannian manifold. Let $u(x, t)$ be a non-negative, bounded, weak solution of

$$
u_{t}=\triangle_{p} u
$$

on $M \times[0, T)$ where $p>2$ and $T>0$, and suppose that for some ball $B\left(x_{0}, d\right)$

$$
B\left(x_{0}, d\right) \cap \operatorname{supp} u(\cdot, 0)=\emptyset .
$$

Then, there exists $t_{0}>0$ such that for all $0 \leq t<\min \left(t_{0}, T\right)$,

$$
B\left(x_{0}, \frac{d}{2}\right) \cap \operatorname{supp} u(\cdot, t)=\emptyset .
$$


The constant $t_{0}$ has the explicit value

$$
t_{0}=C d^{p}\|u(\cdot, 0)\|_{\infty, M}^{-(p-2)}
$$

where $C$ depends on $p$, and on some intrinsic geometric properties of the ball $B\left(x_{0}, d\right)$; in particular, it can be estimated in terms of bounds on the Ricci curvature on $B\left(x_{0}, d\right)$ (see Theorem 4.1 for more details).

The proof of this theorem (see the proof of Theorem 4.1 in Section 4) uses a mean value type inequality, which generalises a well known result for solutions of the heat equation. Two versions of the inequality are discussed in Section 3. We emphasise that the proofs only depend on a local analogue of the Sobolev inequality in $\mathbb{R}^{n}$, not on the global geometry of $M$.

The preceding theorem can be used to prove that solutions of the parabolic $p$-Laplace equation have finite propagation speed, at least locally (in time), as is stated in the following theorem (cf. Theorem 4.2):

Theorem 1.2. Let $M$ be a non-compact, complete Riemannian manifold, and let $u(x, t)$ be a non-negative, bounded, weak solution of

$$
\left\{\begin{array}{l}
u_{t}=\triangle_{p} u \\
u(\cdot, 0)=u_{0}(\cdot)
\end{array}\right.
$$

on $M \times \mathbb{R}^{+}$, where $\operatorname{supp} u_{0}$ is compact.

There exist $T>0$ and an increasing, non-negative function

$$
r:[0, T) \rightarrow[0,+\infty)
$$

such that for any $0<t<T$, the support of $u(\cdot, t)$ is contained in the $r(t)$ neighbourhood of $\operatorname{supp} u_{0}$.

For the constant $T>0$, we can take

$$
T=\sup _{r>0} C(\nu, p, r) r^{p}\left\|u_{0}\right\|_{\infty, M}^{-(p-2)}
$$

where $C(\nu, p, r)$ depends on volume doubling and Poincaré inequalities in an $r$-neighbourhood of $\operatorname{supp} u_{0}$, and can be estimated in terms of a lower bound on the Ricci curvature on this neighbourhood.

For a proof of this theorem, see the proof of Theorem 4.2 in Section 4. Given a lower bound for the Ricci curvature on the manifold $M$, the results in the previous theorem admit the following global version (cf. Corollary 4.3): 
Corollary 1.3. Let $M$ be a non-compact, complete Riemannian manifold with metric $g$, and let $u$ be a non-negative, bounded, weak solution of

$$
\left\{\begin{array}{l}
u_{t}=\triangle_{p} u \\
u(\cdot, 0)=u_{0}(\cdot)
\end{array}\right.
$$

on $M \times \mathbb{R}^{+}$.

If $\operatorname{supp} u_{0}$ is compact, and the Ricci curvature of $M$ satisfies for all $x \notin \operatorname{supp} u_{0}$

$$
\operatorname{Ric}_{M}(x) \geq-\frac{c(n-1)}{\operatorname{dist}\left(x, \operatorname{supp} u_{0}\right)^{2}} g,
$$

then for all $t>0, \operatorname{supp} u(\cdot, t)$ is contained in the $r(t)$ neighbourhood of $\operatorname{supp} u_{0}$, with

$$
r(t)=C\left(\left\|u_{0}\right\|_{\infty, M}^{p-2} t\right)^{\frac{1}{p}} .
$$

\section{Preliminaries.}

\subsection{Notation.}

Throughout this paper, $M$ will be an $n$-dimensional $(n \geq 2)$, complete, non-compact Riemannian manifold with metric $g$. For every $x \in M, T_{x} M$ denotes the tangent space to $M$ at $x$. The inner product in $T_{x} M$ is denoted by $\langle\cdot, \cdot\rangle$, suppressing the dependence on $x$. If $\sigma$ is a smooth, positive function on $M$, we can define a measure $\mu$ on $M$ by

$$
d \mu=d \mu(x)=\sigma(x) d \nu(x)
$$

where $d \nu$ is the Riemannian measure on $M$. The pair $(M, \mu)$ is called a weighted manifold.

The requirement that we should be able to integrate by parts on $(M, \mu)$ leads to a natural definition of the divergence $\operatorname{div}_{\mu}$ on $(M, \mu)$. In local coordinates,

$$
\operatorname{div}_{\mu} v=\frac{1}{\sigma \sqrt{g}} \frac{\partial}{\partial x_{i}}\left(\sigma \sqrt{g} v^{i}\right)
$$

where $g=\operatorname{det} g_{i j}$, and $g_{i j}$ are the components of the metric tensor. Here, we sum over repeated indices.

Throughout this paper, $\Omega$ will be an open subset of $M$ (not necessarily bounded), $x_{0}$ will be a fixed point in $M$, and $U$ will be a precompact set containing $x_{0}$. We write $I_{T}$ to denote the interval $[0, T)$, where $T>0$ is an arbitrary real number, and $\Omega_{T}$ for $\Omega \times I_{T}$. 
For the geodesic ball of radius $r$ centred at the point $x$, we write $B(x, r)$, and $V(x, r)=\mu(B(x, r))$.

If $f=f(x, t)$ is a function defined on $\Omega_{T}$, we use $\nabla f$ to denote the gradient with respect to the spatial variable $x$. The derivative with respect to $t$ is denoted $f_{t}$.

Also, we write

$$
f_{+}=\max (f, 0) .
$$

If $f \in L^{q}(\Omega)=L^{q}(\Omega ; \mu)$, then $\|f\|_{q, \Omega}$ denotes the $L^{q}(\Omega ; \mu)$ norm of $f$. For $q \geq 1$ the spaces $W^{1, q}(\Omega)=W^{1, q}(\Omega ; \mu)$ and $\stackrel{o}{W^{1, p}}(\Omega)=\stackrel{o}{W}{ }^{1, p}(\Omega ; \mu)$ are the usual Sobolev spaces.

The notation $f \in L^{q}\left(I_{T} ; L^{q}(\Omega)\right)$ means that $f$ is a function defined on $\Omega_{T}$ such that for almost every $t \in I_{T}$ the function $x \mapsto f(x, t)$ is in $L^{q}(\Omega)$, and

$$
\int_{I_{T}} \int_{\Omega}|f|^{q} d \mu d t<\infty
$$

The function spaces $L^{q}\left(I_{T} ; W^{1, q}(\Omega)\right), L^{q}\left(I_{T} ; \stackrel{o}{W^{1, q}}(\Omega)\right), C^{k}\left(I_{T} ; L^{q}(\Omega)\right)$ and $W^{1, q}\left(I_{T} ; L^{q}(\Omega)\right)$ are defined analogously.

\subsection{Degenerate parabolic equations and weak solutions.}

We consider the following parabolic equation on $M$,

$$
u_{t}=\operatorname{div}_{\mu} \mathcal{A}(x, t, u, \nabla u)
$$

where $u=u(x, t), x \in M, t \geq 0$.

Throughout this paper, we assume that the mapping $\mathcal{A}: M \times \mathbb{R}^{+} \times$ $\mathbb{R} \times T M \rightarrow T M$ is such that $\mathcal{A}(x, t, u, \xi)$ is measurable in $(x, t, u, \xi)$ and continuous in $(u, \xi)$ for a.e. $(x, t) \in M \times \mathbb{R}^{+}$and that $\mathcal{A}$ satisfies the following conditions for real constants

$$
2<p<\infty \text {, and } c, C>0 \text { : }
$$

for all $x \in M, t \in \mathbb{R}^{+}, u \in \mathbb{R}$ and $\xi \in T_{x} M$

$$
\begin{gathered}
|\mathcal{A}(x, t, u, \xi)| \leq c|\xi|^{p-1} \\
\langle\mathcal{A}(x, t, u, \xi), \xi\rangle \geq C|\xi|^{p}
\end{gathered}
$$

Note that, by (2.2), Equation (2.1) degenerates if $\nabla u=0$. 
Solutions of $(2.1)$ on a set $\Omega_{T}$ are defined as follows: a function $u(x, t)$ is a weak sub-solution resp. super-solution of (2.1) on $\Omega_{T}$ if

$$
u \in \mathcal{S}_{p}\left(\Omega_{T}\right)=C\left(I_{T} ; L^{2}(\Omega)\right) \cap L^{p}\left(I_{T} ; W^{1, p}(\Omega)\right)
$$

and for all $t_{1}, t_{2} \in I_{T}, t_{1}<t_{2}$, for all non-negative test functions

$$
\phi \in \mathcal{T}_{p}\left(\Omega_{T}\right)=W^{1,2}\left(I_{T} ; L^{2}(\Omega)\right) \cap L^{p}\left(I_{T} ; \stackrel{o}{W^{1, p}}(\Omega)\right)
$$

$u$ satisfies

$$
\left.\int_{\Omega} u \phi d \mu\right|_{t=t_{1}} ^{t_{2}}+\int_{t_{1}}^{t_{2}} \int_{\Omega}\left[-u \phi_{t}+\langle\mathcal{A}(x, t, u, \nabla u), \nabla \phi\rangle\right] d \mu d t \leq(\text { resp. } \geq) 0 .
$$

A function that is both a weak sub-solution and a weak super-solution is a weak solution.

If we assume that in addition to $(2.2)$ and $(2.3), \mathcal{A}$ satisfies

$$
\left\langle\mathcal{A}\left(x, t, u, \xi_{1}\right)-\mathcal{A}\left(x, t, u, \xi_{2}\right), \xi_{1}-\xi_{2}\right\rangle \geq 0,
$$

for all $(x, t, u) \in M \times \mathbb{R}^{+} \times \mathbb{R}$ and for all $\xi_{1}, \xi_{2} \in T_{x} M$, as is clearly the case for the parabolic $p$-Laplace equation, and that $\Omega$ is bounded, then (see [16, chapter V]) a Galerkin procedure can be used to show that the Dirichlet problem

$$
\begin{cases}u_{t}=\operatorname{div}_{\mu} \mathcal{A}(x, t, u, \nabla u) & \text { in } \Omega_{T}, \\ \left.u\right|_{\partial \Omega \times(0, T)}=0, & \\ u(x, 0)=u_{0}(x), & u_{0} \in L^{2}(\Omega),\end{cases}
$$

has a weak solution, by which we mean a solution to (2.1) that is in

$$
\stackrel{o}{\mathcal{S}}_{p}\left(\Omega_{T}\right)=C\left(I_{T} ; L^{2}(\Omega)\right) \cap L^{p}\left(I_{T} ; \stackrel{o}{W^{1, p}}(\Omega)\right),
$$

the class of weak solutions that are zero on $\partial \Omega \times I_{T}$, and satisfies the initial condition.

In the proofs of several of the estimates that will follow, it would have been convenient if in the definition (2.6) of a weak solution, we could have taken $u$ itself (or $u$ multiplied by a cut-off function) as a test function. Unfortunately, as can be seen from the Definitions (2.4) and (2.5), a weak solution of (2.1) is not in general admissible as a test function, since it is not sufficiently regular in $t: u_{t}$ generally only has a meaning in the sense of distributions. This difficulty can be overcome by using the so-called Steklov average of $u$ (see for example [16, Chapter 2]), which is defined as follows: 
Definition 2.1. Let $u$ be a measurable function on $\Omega_{T}$. For $h \in(0, T)$, define the Steklov average $u_{h}$ on $\Omega_{T}$ by

$$
u_{h}(\cdot, t)= \begin{cases}\frac{1}{h} \int_{t}^{t+h} u(\cdot, \tau) d \tau, & t \in I_{T-h} \\ 0, & t \geq T-h\end{cases}
$$

If $u$ is a sub- resp. super-solution of $(2.1)$ in $\Omega_{T}$, then the Steklov average $u_{h}$ is in $C^{1}\left(I_{T-h} ; L^{2}(\Omega)\right)$ and satisfies

$$
\int_{\Omega}\left[\frac{\partial u_{h}(x, t)}{\partial t} \phi(x)+\left\langle[\mathcal{A}(x, t, u, \nabla u)]_{h}(x, t), \nabla \phi(x)\right\rangle\right] d \mu(x) \leq(\text { resp. } \geq) 0,
$$

$\forall h \in(0, T), \forall t \in I_{T-h}, \forall \phi \in L^{2}(\Omega) \cap \stackrel{o}{W^{1, p}}(\Omega)$. In contrast to the situation for the non-averaged function $u, u_{h}$ can be used as a test function in (2.9). We will use this in combination with the following standard lemma, which can easily be proved using Hölder's inequality,

Lemma 2.2. If $u \in C\left(I_{T} ; L^{q}(\Omega)\right)$, then $u_{h}(\cdot, t) \rightarrow u(\cdot, t)$ in $L^{q}(\Omega)$ as $h \rightarrow 0$ for every $t \in I_{T}$. If $u \in L^{q}\left(\Omega_{T}\right)$, then $u_{h} \rightarrow u$ in $L^{q}\left(\Omega_{T}\right)$ as $h \rightarrow 0$.

In this paper, we consider only bounded solutions of (2.1) and (2.7). Using Steklov averages, one can prove that a sufficient condition for a solution $u \in \stackrel{o}{\mathcal{S}}_{p}\left(\Omega_{T}\right)$ of the Dirichlet problem (2.7) to be bounded is that $u_{0}$ is bounded. To show this, we use the following lemma that is proved in $[8$, Chapter II]:

Lemma 2.3 ([8]). If $u$ is a sub-solution of (2.1) in $\Omega_{T}$, and $\mathcal{A}$ satisfies (2.3), then for any $\theta \in \mathbb{R}^{+}$

$$
(u-\theta)_{+}
$$

is a sub-solution of (2.1) in $\Omega_{T}$.

We use this lemma to prove

Lemma 2.4. Let $u \in \stackrel{o}{\mathcal{S}}_{p}\left(\Omega_{T}\right)$ be a sub-solution of (2.7). If $\mathcal{A}$ satisfies (2.3) and $u_{0} \in L^{\infty}(\Omega)$, then $u(\cdot, t) \in L^{\infty}(\Omega)$ for all $t \in I_{T}$, and

$$
\|u(\cdot, t)\|_{\infty, \Omega} \leq\left\|u_{0}\right\|_{\infty, \Omega} .
$$

Proof. If we define

$$
v=\left(u-\left\|u_{0}\right\|_{\infty, \Omega}\right)
$$


then, by Lemma $2.3, v_{+}$is a subsolution of (2.7), so its Steklov average satisfies (2.9) $\forall h \in(0, T), \forall t \in I_{T-h}, \forall \phi \in L^{2}(\Omega) \cap \stackrel{o}{W^{1, p}}(\Omega)$. If $t \in I_{T}$ and $h \in(0, T-t)$, then

$$
\left[\left(u-\left\|u_{0}\right\|_{\infty, \Omega}\right)_{+}\right]_{h}(\cdot, \tau) \in L^{2}(\Omega) \cap \stackrel{o}{W^{1, p}}(\Omega),
$$

so it is a valid test function in (2.9). Integrating (2.9) over $[0, t], t \in I_{T}$, with this choice of test function gives

$$
\begin{aligned}
& \frac{1}{2} \int_{\Omega}\left(\left[v_{+}\right]_{h}\right)^{2}(x, t) d \mu(x)-\frac{1}{2} \int_{\Omega}\left(\left[v_{+}\right]_{h}\right)^{2}(x, 0) d \mu(x) \\
& \quad \leq-\int_{0}^{t} \int_{\Omega}\left\langle\left[\mathcal{A}\left(x, t, v_{+}, \nabla v_{+}\right)\right]_{h}, \nabla\left[v_{+}\right]_{h}(x, \tau)\right\rangle d \mu(x) d \tau
\end{aligned}
$$

Letting $h \rightarrow 0$, and applying Lemma 2.2, we find

$$
\begin{aligned}
\int_{\Omega}\left(u-\left\|u_{0}\right\|_{\infty, \Omega}\right)_{+}^{2} & (x, t) d \mu(x) \\
& \leq-\int_{0}^{t} \int_{\Omega}\left\langle\mathcal{A}\left(x, t, v_{+}, \nabla v_{+}\right), \nabla v_{+}(x, \tau)\right\rangle d \mu(x) d \tau \\
& \leq 0
\end{aligned}
$$

by the estimate $(2.3)$, for all $t \in I_{T}$.

\subsection{Local Sobolev inequality.}

The proof in $[9,14]$ of the finite propagation speed property for solutions of (2.1) relies on the Sobolev inequality in $\mathbb{R}^{n}$. On a manifold $M$, a Sobolev inequality in general does not hold, see for example [20]. However, a local version does hold on any given Riemannian manifold $M$. Recall that $U \subset$ $M$ is a precompact set. By a compactness argument, the following two conditions are always satisfied. First, there exist positive constants $D_{U}$ and $\nu$ such that for all balls

$$
B\left(x, r_{1}\right), B\left(y, r_{2}\right) \subset U,
$$

with $r_{1} \leq r_{2}$, the following volume doubling inequality holds

$$
\frac{V\left(y, r_{2}\right)}{V\left(x, r_{1}\right)} \leq D_{U}\left(\frac{r_{2}}{r_{1}}\right)^{\nu}
$$


Here, $\nu$ can be taken equal to or larger than the dimension $n$ of the manifold, so we can assume

$$
\nu>p .
$$

Second, the following Poincaré inequality holds: there exists a positive constant $P_{U}$ such that if $B(x, r) \subset U$ is a ball split by a hyper-surface $\Gamma$ into two disjoint parts $U_{1}$ and $U_{2}$ such that $B(x, r) \backslash \Gamma=U_{1} \cup U_{2}$, then

$$
P_{U} A(\Gamma) \geq \frac{1}{r} \min \left(\mu\left(U_{1}\right), \mu\left(U_{2}\right)\right)
$$

where $A(\Gamma)$ is the $(n-1)$-dimensional measure of $\Gamma$, weighted by $\sigma$.

It can be proved from (2.10) and (2.11) (following a procedure similar to that in [10] or [19], see [6]) that if $M$ is non-compact, for any ball $B(x, r) \subset$ $U$, for all non-negative $f \in \stackrel{o}{W^{1, p}}(B(x, r))$

$$
\left(\int_{B(x, r)} f^{\frac{p \nu}{\nu-p}} d \mu\right)^{\frac{\nu-p}{\nu}} \leq S_{x, r, U} \int_{B(x, r)}|\nabla f|^{p} d \mu
$$

with

$$
S_{x, r, U}=C(\nu, p)\left(D_{U}^{2} P_{U}\right)^{p} \frac{r^{p}}{V(x, r)^{\frac{p}{\nu}}} .
$$

This local version of the Sobolev inequality is one of the tools that we will be using in later proofs.

We will mostly be using these inequalities in balls $U=B\left(x_{0}, r\right) \subset \Omega$, where $x_{0}$ is fixed, in which case, we will use the shorter notation

$$
P=P_{B\left(x_{0}, r\right)}, \quad D=D_{B\left(x_{0}, r\right)}, \quad S_{r}=S_{x_{0}, r, B\left(x_{0}, r\right)}
$$

for the constants in (2.13).

The constants $P_{U}$ and $D_{U}$ both are curvature dependent, and can be estimated in terms of bounds on the Ricci curvature. Since the Ricci curvature of $M$ is a bilinear form, we can compare it with the metric $g$. If $\mu$ is the Riemannian measure on $M, U=B\left(x_{0}, r\right)$ and the Ricci curvature on $B\left(x_{0}, r\right)$ is bounded from below by

$$
-(n-1) k g
$$

$k>0$, then the constants $D$ and $P$ satisfy

$$
D, P \leq C e^{C_{n} \sqrt{k} r}
$$


see [12], resp. [10, 5]. If the manifold $M$ has non-negative Ricci curvature, the constants $D_{U}$ and $P_{U}$ are global.

Most of the estimates that we will obtain are curvature dependent, as a consequence of the curvature dependence through $D_{U}$ and $P_{U}$ of the constant $S_{x, r, U}$ in (2.12).

In this paper, we assume that $M$ is non-compact. If $M$ is compact, then provided that $\overline{B(x, r)} \neq M,(2.12)$ holds with the constant $S_{x, r, U}$ replaced with

$S_{x, r, M}=C(\nu, p)\left(\frac{1}{\min (V(x, r), \mu(M)-V(x, r))}\right)^{p}\left(D_{M} P_{M}\right)^{p}\left(\frac{\mu(M)^{1-\frac{1}{\nu}}}{\operatorname{diam} M}\right)^{p}$,

where $\operatorname{diam} M$ is the diameter of the manifold (see [6]). All proofs in this paper can easily be modified to apply to compact manifolds if in all references to the local Sobolev inequality (2.15) is used instead of (2.13).

\section{Mean value type inequalities.}

In this section, we assume

$$
p \geq 2 .
$$

If $M$ is a geodesically complete manifold of non-negative Ricci curvature, a positive solution $u$ of the heat equation in a cylinder $B(x, \sqrt{t}) \times(0, t]$, $x \in M, t>0$, is known to satisfy the following mean value inequality, see for example [11]:

$$
u(x, t) \leq C \frac{1}{t V(x, \sqrt{t})} \int_{0}^{t} \int_{B(x, \sqrt{t})} u d \mu d \tau,
$$

We will show that this is a special case of an estimate that holds for subsolutions of Equation (2.1). In the second half of this section, we show that for $t>0$ a simplified mean value type inequality holds away from the support of $u(\cdot, 0)$. The result we obtain in this case will be used to prove that solutions to (2.1) have finite propagation speed.

We start with a preliminary lemma, giving an estimate for sub-solutions of (2.1).

Lemma 3.1. Let $u \in \mathcal{S}_{p}\left(\Omega_{T}\right)$ be a bounded, non-negative, weak subsolution of (2.1), where the mapping $\mathcal{A}$ is assumed to satisfy (2.2) and (2.3). Let $\eta$ be a piecewise smooth, bounded, non-negative function in $\Omega_{T}$, with 
compact support in $\Omega$ at all times $t \in I_{T}$, and with bounded first order derivatives, and let $q \geq 2, s \geq p$. Fix $\theta \geq 0$ and denote $v=(u-\theta)_{+}$. Then,

$$
\begin{aligned}
& \left.\int_{\Omega} v^{q} \eta^{s} d \mu\right|_{t_{1}} ^{t_{2}}+\int_{t_{1}}^{t_{2}} \int_{\Omega}\left|\nabla\left(v^{\frac{p+q-2}{p}}\right)\right|^{p} \eta^{s} d \mu d \tau \\
& \quad \leq C\left(\|v\|_{\infty, \Omega_{T}}^{p-2}\|\nabla \eta\|_{\infty, \Omega_{T}}^{p}\|\eta\|_{\infty, \Omega_{T}}^{s-p}+\|\eta\|_{\infty, \Omega_{T}}^{s-1}\left\|\eta_{t}\right\|_{\infty, \Omega_{T}}\right) \int_{t_{1}}^{t_{2}} \int_{\Omega} v^{q} d \mu d \tau,
\end{aligned}
$$

for all $0<t_{1}<t_{2}<T$, with $C=C(\mathcal{A}, q, s)$.

Proof. By Lemma 2.3, $v$ is a sub-solution of (2.1), so its Steklov average satisfies

$$
\int_{\Omega}\left[\frac{\partial v_{h}(x, t)}{\partial t} \phi(x)+\left\langle[\mathcal{A}(x, t, v, \nabla v)]_{h}(x, t), \nabla \phi(x)\right\rangle\right] d \mu(x) \leq 0
$$

$\forall h \in(0, T), \forall t \in I_{T-h}, \forall \phi \in L^{2}(\Omega) \cap \stackrel{o}{W^{1, p}}(\Omega)$. By assumption, $u$ is bounded, so for fixed $t \in I_{T-h}$

$$
\left(v_{h}\right)^{q-1}(\cdot, t) \eta^{s} \in L^{2}(\Omega) \cap \stackrel{o}{W^{1, p}}(\Omega),
$$

which makes it a valid test function in (3.2). Integrating the equation over a time interval $\left[t_{1}, t_{2}\right]$, we get

$$
\begin{aligned}
\left.\int_{\Omega}\left[v_{h}\right]^{q} \eta^{s} d \mu\right|_{t_{1}} ^{t_{2}} & \\
& \leq \int_{t_{1}}^{t_{2}} \int_{\Omega}\left[-q\left\langle[\mathcal{A}(x, t, v, \nabla v)]_{h}, \nabla\left[\left(v_{h}\right)^{q-1} \eta^{s}\right]\right\rangle+s\left[v_{h}\right]^{q} \eta^{s-1} \eta_{t}\right] d \mu d t .
\end{aligned}
$$

Now, let $h \rightarrow 0$. Since $u \in \mathcal{S}_{p}\left(\Omega_{T}\right)$ is bounded and $\mathcal{A}$ satisfies (2.2), all Steklov averages in (3.3) converge to the corresponding non-averaged functions in the appropriate spaces by Lemma 2.2. Applying the estimates (2.2) and (2.3) for $\mathcal{A}$ as well as Young's inequality, we get

$$
\begin{aligned}
&\left.\int_{\Omega} v^{q} \eta^{s} d \mu\right|_{t_{1}} ^{t_{2}} \leq \int_{t_{1}}^{t_{2}} \int_{\Omega}\left[-C q(q-1) v^{q-2} \eta^{s}|\nabla v|^{p}+s v^{q} \eta^{s-1} \eta_{t}\right. \\
&\left.+c s q v^{q-1} \eta^{s-1}|\nabla v|^{p-1}|\nabla \eta|\right] d \mu d \tau \\
& \leq A \int_{t_{1}}^{t_{2}} \int_{\Omega}\left[-\left|\nabla v^{\frac{p+q-2}{p}}\right|^{p} \eta^{s}+s v^{q} \eta^{s-1} \eta_{t}+|\nabla \eta|^{p} \eta^{s-p} v^{p+q-2}\right] d \mu d \tau
\end{aligned}
$$


for a constant $A=A(c, C, q, s)>0$. The lemma now follows.

The restriction that $u$ is bounded is not necessary if $q=2$.

The next step toward our mean value inequality involves a comparison of integrals of a sub-solution of (2.1) over two cylinders, one contained in the other, of $(u-\theta)_{+}^{q}$, with $\theta$ a positive constant and $q \geq 2$, using a local Sobolev inequality in a precompact set $U$. This estimate will later be used repeatedly for a sequence of shrinking cylinders.

We define

$$
\kappa=\kappa(q)=q p+\nu(p-2)
$$

where $\nu$ is the constant in (2.10).

The following is a generalisation of a result in [9].

Lemma 3.2. Let $r_{0}>0$, define $U=B\left(x_{0}, r_{0}\right)$ and let $0<r_{1}<r_{0}, 0 \leq$ $t_{0}<t_{1}<T, 0 \leq \theta_{0}<\theta_{1}$. Define two cylinders

$$
\Psi_{i}=B\left(x_{0}, r_{i}\right) \times\left[t_{i}, T\right), \quad i=0,1 .
$$

Let $u \in \mathcal{S}_{p}\left(\Psi_{0}\right)$ be a non-negative, bounded, weak sub-solution of Equation (2.1). The mapping $\mathcal{A}$ is assumed to satisfy (2.2) and (2.3). Define two integrals

$$
Y_{i}=\iint_{\Psi_{i}}\left(u-\theta_{i}\right)_{+}^{q} d \mu d \tau, \quad i=0,1
$$

Denote $\delta_{r}=r_{0}-r_{1}, \delta_{\theta}=\theta_{1}-\theta_{0}$ and $\delta_{t}=t_{1}-t_{0}$. If $q \geq 2$, then

$$
Y_{1} \leq C \delta_{\theta}^{-\frac{q \kappa}{\kappa+q \nu}} S_{r_{0}}^{\frac{q \nu}{\kappa+q \nu}}\left[\delta_{r}^{-p}\|u\|_{\infty, \Psi_{0}}^{p-2}+\delta_{t}^{-1}\right]^{\frac{q(\nu+p)}{\kappa+q \nu}} Y_{0}^{1+\frac{p q}{\kappa+q \nu}}
$$

The constant $\kappa$ was defined in (3.4), $S_{r_{0}}$ in (2.12) and $\nu$ in (2.10), and $C$ depends on $p, q$ and $\nu$.

Proof.

Write $v=\left(u-\theta_{1}\right)_{+}$. By Hölder's inequality, for all $l>q$,

$$
Y_{1} \leq\left(\iint_{\Psi_{1}} v^{l} d \mu d \tau\right)^{\frac{q}{l}} \mu\left(\left\{(x, t) \in \Psi_{1} \mid u(x, t)>\theta_{1}\right\}\right)^{1-\frac{q}{l}} .
$$


The second term on the right-hand side of (3.5) can easily be estimated in terms of $Y_{0}$ : since $\Psi_{1} \subset \Psi_{0}$, and $\theta_{1}>\theta_{0}$

$$
\begin{aligned}
\mu\left(\left\{(x, t) \in \Psi_{1} \mid u(x, t)>\theta_{1}\right\}\right) & \leq \iint_{\Psi_{0}} \frac{\left(u-\theta_{0}\right)_{+}^{q}}{\left(\delta_{\theta}\right)^{q}} 1_{\left\{u>\theta_{1}\right\}} d \mu d \tau \\
& \leq\left(\delta_{\theta}\right)^{-q} Y_{0} .
\end{aligned}
$$

To estimate the integral on the right-hand side of (3.5), introduce $\tilde{r}_{0}=$ $\frac{r_{0}+r_{1}}{2}$, and let $\zeta(x, t) \equiv \zeta(x)$ be a piecewise smooth, non-negative function supported in $B\left(x_{0}, \tilde{r}_{0}\right)$, such that $\zeta \equiv 1$ on $B\left(x_{0}, r_{1}\right),|\nabla \zeta| \leq \frac{2}{\delta_{r}}$ and $\zeta \leq 1$ on $B\left(x_{0}, \tilde{r}_{0}\right)$. Then, using Hölder's inequality,

$$
\begin{aligned}
\iint_{\Psi_{1}} v^{l} d \mu d \tau \leq & \int_{t_{1}}^{T} \int_{B\left(x_{0}, \tilde{r}_{0}\right)} v^{l} \zeta^{p} d \mu d \tau \\
\leq & \sup _{t_{1} \leq \tau<T}\left(\int_{B\left(x_{0}, \tilde{r}_{0}\right)} v^{\frac{(l-p-q+2) \nu}{p}} d \mu\right)^{\frac{p}{\nu}} \times \\
& \times \int_{t_{1}}^{T}\left(\int_{B\left(x_{0}, \tilde{r}_{0}\right)}\left(v^{\frac{p+q-2}{p}} \zeta\right)^{\frac{p \nu}{\nu-p}} d \mu\right)^{\frac{\nu-p}{\nu}} d \tau
\end{aligned}
$$

provided that $l$ satisfies

$$
l>p+q-2 .
$$

This replaces the earlier condition $l>q$.

Since $u$ is bounded, $v^{\frac{p+q-2}{p}} \zeta \in L^{p}\left(I_{T} ; \stackrel{o}{W^{1, p}}\left(B\left(x_{0}, \tilde{r}_{0}\right)\right)\right)$, so we can apply the local Sobolev inequality (2.12):

$$
\begin{aligned}
& \int_{t_{1}}^{T}\left(\int_{B\left(x_{0}, \tilde{r}_{0}\right)}\left(v^{\frac{p+q-2}{p}} \zeta d \mu\right)^{\frac{p \nu}{\nu-p}} d \mu\right)^{\frac{\nu-p}{\nu}} d \tau \\
& \quad \leq S_{r_{0}} \int_{t_{1}}^{T} \int_{B\left(x_{0}, \tilde{r}_{0}\right)}\left|\nabla\left(v^{\frac{p+q-2}{p}} \zeta\right)\right|^{p} d \mu d \tau \\
& \quad \leq S_{r_{0}} C \int_{t_{1}}^{T} \int_{B\left(x_{0}, \tilde{r}_{0}\right)}\left[\left|\nabla v^{\frac{p+q-2}{p}}\right|^{p}+\left(\frac{2}{\delta_{r}}\right)^{p} v^{p+q-2}\right] d \mu d \tau .
\end{aligned}
$$

Substituting (3.9) into (3.7), we get

$$
\begin{array}{rl}
\iint_{\Psi_{1}} v^{l} & d \mu d \tau \leq C S_{r_{0}} \sup _{t_{1} \leq \tau<T}\left(\int_{B\left(x_{0}, \tilde{r}_{0}\right)} v^{\frac{(l-p-q+2) \nu}{p}} d \mu\right)^{\frac{p}{\nu}} \times \\
& \times \int_{t_{1}}^{T} \int_{B\left(x_{0}, \tilde{r}_{0}\right)}\left[\left|\nabla\left(v^{\frac{p+q-2}{p}}\right)\right|^{p}+\left(\frac{2}{\delta_{r}}\right)^{p}\|v\|_{\infty, \Psi_{0}}^{p-2} v^{q}\right] d \mu d \tau .
\end{array}
$$


To estimate this in terms of $Y_{0}$, we apply Lemma 3.1 in $B\left(x_{0}, r_{0}\right) \times I_{T}$, which yields estimates for the integrals on the right-hand side of (3.10).

For the function $\eta$ in Lemma 3.1, we take $\eta(x, \tau)=\eta_{1}(x) \eta_{2}(\tau)$, with $\eta_{1}$ a piecewise smooth, non-negative function supported in $B\left(x_{0}, r_{0}\right)$ such that $\eta_{1} \equiv 1$ on $B\left(x_{0}, \tilde{r}_{0}\right),\left|\nabla \eta_{1}\right| \leq \frac{2}{\delta_{r}}, \eta_{1} \leq 1$ on $B\left(x_{0}, r_{0}\right)$, and

$$
\eta_{2}(\tau)= \begin{cases}\frac{\tau-t_{0}}{\delta_{t}} & t_{0} \leq \tau<t_{1} \\ 1 & t_{1} \leq \tau<T\end{cases}
$$

Lemma 3.1, with $s=p$, now gives that for any $t_{1} \leq \tau<T$ (note that $\left.\eta\left(\cdot, t_{0}\right) \equiv 0\right)$

$$
\begin{aligned}
\int_{B\left(x_{0}, \tilde{r}_{0}\right)} v^{q}(x, \tau) d \mu(x) & +\int_{t_{0}}^{\tau} \int_{B\left(x_{0}, \tilde{r_{0}}\right)}\left|\nabla\left(v^{\frac{p+q-2}{p}}(x, t)\right)\right|^{p} d \mu(x) d t \\
& \leq C\left(\delta_{r}^{-p}\|v\|_{\infty, \Psi_{0}}^{p-2}+\delta_{t}^{-1}\right) Y_{0} .
\end{aligned}
$$

This provides us with the necessary estimate for (3.10), if we choose $l$ so that

$$
\frac{l-p-q+2}{p} \nu=q \text {, i.e. } l=\frac{\kappa+q \nu}{\nu} .
$$

Observe that $l$ satisfies the Condition (3.8). We can now rewrite (3.10)

$$
\iint_{\Psi_{1}} v^{l} d \mu d \tau \leq C S_{r_{0}}\left(\delta_{r}^{-p}\|u\|_{\infty, \Psi_{0}}^{p-2}+\delta_{t}^{-1}\right)^{1+\frac{p}{\nu}} Y_{0}^{1+\frac{p}{\nu}}
$$

To finish the proof of the lemma, we substitute this and (3.6) into (3.5).

The following theorem contains the first of two mean value type inequalities. The proof consists of two steps. In the first step, taking ideas from [9], we repeatedly apply the last lemma in a sequence of shrinking cylinders, whereas in the second step, we apply a technique from [17] that requires growing cylinders.

Theorem 3.3. Assume that $(M, \mu)$ is a non-compact, complete, weighted Riemannian manifold.

Let $r>0, T>0$, and define $U=B\left(x_{0}, r\right)$ and

$$
\Psi_{0}=B\left(x_{0}, r\right) \times I_{T}, \quad \Psi=B\left(x_{0}, \frac{r}{2}\right) \times\left[\left(\frac{1}{2}\right)^{p} T, T\right) .
$$


Let $u \in \mathcal{S}_{p}\left(\Psi_{0}\right)$ be a non-negative, bounded, weak sub-solution of (2.1), where $\mathcal{A}$ is assumed to satisfy the estimates (2.2) and (2.3). Then, for $q \geq 1$

$$
\|u\|_{\infty, \Psi} \leq C S_{r}^{\frac{\nu}{\kappa}}\left(\iint_{\Psi_{0}} u^{q} d \mu d \tau\right)^{\frac{p}{\kappa}}\left[\left(r^{-p}\|u\|_{\infty, \Psi_{0}}^{p-2}+T^{-1}\right)\right]^{\frac{\nu+p}{\kappa}}
$$

$C=C(\nu, p, q), \nu$ and $S_{r}$ as in (2.10) and (2.12), and $\kappa$ given by (3.4).

\section{Proof.}

Step 1: We will first prove the theorem for $q \geq 2$. To do this, we follow ideas from [9], and define a sequence of increasing times $t_{m}$ and a sequence of decreasing radii $r_{m}$

$$
\begin{aligned}
r_{m} & =\frac{1}{2}\left(1+\left(\frac{1}{2}\right)^{m}\right) r \\
t_{m} & =\left(\frac{1}{2}\right)^{p}\left(1-\left(\frac{1}{2}\right)^{p m}\right) T
\end{aligned}
$$

and a corresponding sequence of shrinking cylinders $\Psi_{m}$ :

$$
\Psi_{m}=B\left(x_{0}, r_{m}\right) \times\left[t_{m}, T\right) .
$$

Note that $\Psi=\lim _{m \rightarrow \infty} \Psi_{m}$, where $\Psi$ was defined in (3.14).

We introduce an increasing sequence $\theta_{m}$,

$$
\theta_{m}=\left(1-\left(\frac{1}{2}\right)^{m}\right) \theta
$$

where a value for $\theta>0$ will be fixed below, and, finally, we introduce the following integrals:

$$
Y_{m}=\iint_{\Psi_{m}}\left(u-\theta_{m}\right)_{+}^{q} d \mu d \tau .
$$

Obviously, the $Y_{m}$ are non-negative and decreasing. With a suitable choice of $\theta$, we can achieve that $\lim _{m \rightarrow \infty} Y_{m}=0$ : by Lemma 3.2

$$
Y_{m+1} \leq a b^{m} Y_{m}^{1+\frac{p q}{\kappa+q \nu}},
$$

with

$$
\begin{aligned}
& a=C\left(S_{r}^{q \nu} \theta^{-q \kappa}\left[\left(r^{-p}\|u\|_{\infty, \Psi_{0}}^{p-2}+T^{-1}\right)\right]^{q(\nu+p)}\right)^{\frac{1}{\kappa+q \nu}} \\
& b=2^{\frac{q \kappa+p q(\nu+p)}{\kappa+q \nu}} .
\end{aligned}
$$


Now, since $b>1$, if $Y_{0} \leq a^{-\frac{\kappa+q \nu}{p q}} b^{-\left(\frac{\kappa+q \nu}{p q}\right)^{2}}$ then, by a standard result,

$$
\lim _{m \rightarrow \infty} Y_{m}=0 .
$$

To satisfy the condition on $Y_{0}$, we choose $\theta$ so that

$$
\theta \geq C Y_{0}^{\frac{p}{\kappa}} S_{r}^{\frac{\nu}{\kappa}}\left[\left(r^{-p}\|u\|_{\infty, \Psi_{0}}^{p-2}+T^{-1}\right)\right]^{\frac{\nu+p}{\kappa}}
$$

With this choice of $\theta$, we obtain $(u(\xi, \tau)-\theta)_{+}=0$ a.e. on $\Psi$, which completes the proof of the theorem for $q \geq 2$.

Step 2: We now extend the proof to include $1 \leq q<2$. Consider $q, l$ such that $1 \leq q<2 \leq l$. We will use the notation

$$
\kappa_{q}=p q+\nu(p-2), \quad \kappa_{l}=p l+\nu(p-2) .
$$

Following ideas from [17], we use a sequence of growing cylinders. Define

$$
\begin{aligned}
& r_{m}=\left(\frac{1}{2}\right)^{m+1} r, \\
& t_{m}=\left(\frac{1}{2}\right)^{p(m+1)} T,
\end{aligned}
$$

and

$$
\tilde{\Psi}_{m}=B\left(x_{0}, r-r_{m}\right) \times\left[t_{m}, T\right) .
$$

Note that $\tilde{\Psi}_{0}=\Psi$ and $\lim _{m \rightarrow \infty} \tilde{\Psi}_{m}=\Psi_{0}$.

Let $(\xi, \tau) \in \tilde{\Psi}_{m-1}$. We now introduce two cylinders containing $(\xi, \tau)$, both of them contained in $\tilde{\Psi}_{m}$, in which we can apply the result from step 1 (see Figure 1): choose $s$ such that

$$
\tau<s<\min \left(\tau+\left(1-\left(\frac{1}{2}\right)^{p}\right) t_{m}, T\right)
$$

and define

$$
\psi_{0}(\xi, \tau):=B\left(\xi, r_{m}\right) \times\left[s-t_{m}, s\right) .
$$

Since $p \geq 2, \psi_{0}(\xi, \tau) \subset \tilde{\Psi}_{m}$. We also define

$$
\psi(\xi, \tau):=B\left(\xi, r_{m+1}\right) \times\left[s-\left(1-\left(\frac{1}{2}\right)^{p}\right) t_{m}, s\right) \subset \psi_{0}(\xi, \tau) .
$$

Observe that $(\xi, \tau) \in \psi(\xi, \tau)$. Since $l \geq 2$, we can apply the result from step 


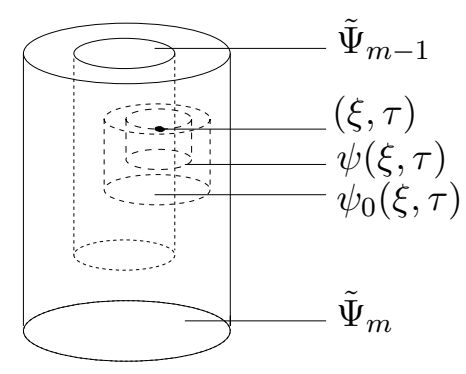

Figure 1: Cylinders $\tilde{\Psi}_{m+1}, \tilde{\Psi}_{m}, \psi$ and $\psi_{0}$

1 in the cylinders $\psi(\xi, \tau)$ and $\psi_{0}(\xi, \tau)$ to conclude that for all $(\xi, \tau) \in \tilde{\Psi}_{m-1}$

$$
\begin{aligned}
\|u\|_{\infty, \psi(\xi, \tau)} & \leq C S_{\xi, r_{m}, U}^{\frac{\nu}{\kappa_{l}}}\left(\iint_{\psi_{0}(\xi, \tau)} u^{l} d \mu d s\right)^{\frac{p}{\kappa_{l}}}\left[r_{m}^{-p}\|u\|_{\infty, \psi_{0}(\xi, \tau)}^{p-2}+t_{m}^{-1}\right]^{\frac{\nu+p}{\kappa_{l}}} \\
& \leq C S_{r}^{\frac{\nu}{\kappa_{l}}}\|u\|_{\infty, \tilde{\Psi}_{m}}^{\frac{p(l-q)}{\kappa_{l}}}\left(\iint_{\Psi_{0}} u^{q} d \mu d s\right)^{\frac{p}{\kappa_{l}}}\left(2^{p m}\left[r^{-p}\|u\|_{\infty, \Psi_{0}}^{p-2}+T^{-1}\right]\right)^{\frac{\nu+p}{\kappa_{l}}} .
\end{aligned}
$$

Introducing

$$
M_{m}=\|u\|_{\infty, \tilde{\Psi}_{m}}
$$

the fact that $(3.17)$ holds for all $(\xi, \tau) \in \tilde{\Psi}_{m-1}$ implies

$$
M_{m-1} \leq 2^{\frac{p m(\nu+p)}{\kappa_{l}}} A M_{m}^{\frac{p(l-q)}{\kappa_{l}}},
$$

where the constant $A$ is given by

$$
A=C S_{r}^{\frac{\nu}{\kappa_{l}}}\left(\iint_{\Psi_{0}} u^{q} d \mu d s\right)^{\frac{p}{\kappa_{l}}}\left(r^{-p}\|u\|_{\infty, \Psi_{0}}^{p-2}+T^{-1}\right)^{\frac{\nu+p}{\kappa_{l}}} .
$$

Repeatedly applying (3.18), we obtain

$$
M_{0} \leq A^{\sum_{i=0}^{m-1}\left(\frac{p(l-q)}{\kappa_{l}}\right)^{i}}\left(2^{\frac{p(\nu+p)}{\kappa_{l}}}\right)^{\sum_{i=0}^{m-1}(i+1)\left(\frac{p(l-q)}{\kappa_{l}}\right)^{i}} M_{m}^{\left(\frac{p(l-q)}{\kappa_{l}}\right)^{m}} .
$$

Letting $m \rightarrow \infty$, we get, since $\frac{p(l-q)}{\kappa_{l}}<1$,

$$
M_{0} \leq C A^{\frac{\kappa_{l}}{\kappa_{q}}} .
$$


It now follows immediately from the definitions of $M_{0}$ and $A$, that

$$
\|u\|_{\infty, \Psi} \leq C S_{r}^{\frac{\nu}{\kappa_{q}}}\left(\iint_{\Psi_{0}} u^{q} d \mu d s\right)^{\frac{p}{\kappa_{q}}}\left(r^{-p}\|u\|_{\infty, \Psi_{0}}^{p-2}+T^{-1}\right)^{\frac{\nu+p}{\kappa_{q}}}
$$

the statement of the theorem.

\subsection{A second mean value inequality.}

In the proof of Lemma 3.2, we introduced a cut-off function $\eta$ that vanished on the bottom of the largest of the two cylinders (see (3.11)). If the support of the solution $u$ at time $t=0$ does not intersect the bottom of this cylinder, however, this becomes unnecessary, and a different mean value type inequality holds.

Theorem 3.4. Assume that $(M, \mu)$ is a non-compact, complete, weighted Riemannian manifold. Choose $r>0$ and define $U=B\left(x_{0}, r\right)$. Let $T>0$ and define

$$
\Psi_{0}=B\left(x_{0}, r\right) \times I_{T}, \quad \Psi=B\left(x_{0}, \frac{r}{2}\right) \times I_{T} .
$$

If $u \in \mathcal{S}_{p}\left(U_{T}\right)$ is a non-negative, bounded, weak sub-solution of (2.1), where $\mathcal{A}$ satisfies (2.2) and (2.3), and $u$ is such that

$$
B\left(x_{0}, r\right) \cap \operatorname{supp} u(\cdot, 0)=\emptyset,
$$

then for $q \geq 1$

$$
\|u\|_{\infty, \Psi} \leq C S_{r}^{\frac{\nu}{\kappa}}\left(r^{-p}\|u\|_{\infty, \Psi_{0}}^{p-2}\right)^{\frac{\nu+p}{\kappa}}\left(\iint_{\Psi_{0}} u^{q} d \mu d \tau\right)^{\frac{p}{\kappa}},
$$

where $C=C(\nu, p, q), S_{r}$ and $\nu$ as in (2.12) and $\kappa$ given by (3.4).

Proof. The proof is very similar to that of the first mean value inequality. Again, for $q \geq 2$, we consider a sequence of shrinking cylinders, but this time they all have the same height. Let

$$
\begin{aligned}
r_{m} & =\frac{1}{2}\left(1+\left(\frac{1}{2}\right)^{m}\right) r, \\
\Psi_{m} & =B\left(x_{0}, r_{m}\right) \times I_{T}, \\
\theta_{m} & =\left(1-\left(\frac{1}{2}\right)^{m}\right) \theta, \\
v_{m} & =\left(u-\theta_{m}\right)_{+}, \\
Y_{m} & =\iint_{\Psi_{m}} v_{m} d \mu d \tau,
\end{aligned}
$$


with $\theta$ to be chosen later.

Follow the proof of Lemma 3.2 to obtain, analogous to (3.5) combined with (3.6), (3.10) and (3.13),

$$
\begin{aligned}
Y_{m+1} & \leq\left(C S_{r} \sup _{0 \leq \tau<T}\left(\int_{B\left(x_{0}, \tilde{r}_{m}\right)} v_{m+1}^{q} d \mu\right)^{\frac{p}{\nu}}\right)^{\frac{q \nu}{\kappa+q \nu}} \\
& \times\left(\int_{0}^{T} \int_{B\left(x_{0}, \tilde{r}_{m}\right)}\left[\left|\nabla v_{m+1}^{\frac{p+q-2}{p}}\right|^{p}+v_{m+1}^{p+q-2}\left(\frac{2^{m+3}}{r}\right)^{p}\right] d \mu d \tau\right)^{\frac{\kappa \nu}{\kappa+q \nu}} \\
& \times\left(\frac{2^{q(m+1)}}{\theta^{q}} Y_{m}\right)^{\frac{\kappa}{\kappa+q \nu}}
\end{aligned}
$$

where

$$
\tilde{r}_{m}=\frac{r_{m}+r_{m+1}}{2} \text {. }
$$

Again, we want to apply Lemma 3.1. In the proof of Lemma 3.2, we used a time-dependent cut-off function (see (3.11)) to obtain the inequality (3.12). Since for all $m, \operatorname{supp} u(\cdot, 0) \cap B\left(x_{0}, r_{m}\right) \subset \operatorname{supp} u(\cdot, 0) \cap B\left(x_{0}, r\right)=\emptyset$, we can this time use time-independent cut-off functions $\eta_{m}(x, t)=\eta_{m}(x)$, supported in $B\left(x_{0}, r_{m}\right)$, such that $\eta_{m} \equiv 1$ on $B\left(x_{0}, \tilde{r}_{m}\right)$, with $\left|\nabla \eta_{m}\right| \leq \frac{2^{m+3}}{r}$. In this case, Lemma 3.1 gives, for all $0<\tau<T$,

$$
\begin{gathered}
\int_{B\left(x_{0}, \tilde{r}_{m}\right)} v_{m+1}^{q}(x, \tau) d \mu(x)+\int_{0}^{\tau} \int_{B\left(x_{0}, \tilde{r}_{m}\right)}\left|\nabla\left(v_{m}^{\frac{p+q-2}{p}}(x, t)\right)\right|^{p} d \mu(x) d t \\
\leq C 2^{m p} r^{-p}\left\|v_{m+1}\right\|_{\infty, \Psi_{0}}^{p-2} Y_{m},
\end{gathered}
$$

which is the analogue of (3.12), and leads to

$$
Y_{m+1} \leq 2^{\frac{m\left(p^{2} q+p q \nu+\kappa q\right)}{\kappa+q \nu}} C\left(S_{r}^{q \nu} \theta^{-\kappa q}\left(r^{-p}\left\|v_{m+1}\right\|_{\infty, \Psi_{0}}^{p-2}\right)^{q(p+\nu)}\right)^{\frac{1}{\kappa+q \nu}} Y_{m}^{1+\frac{p q}{\kappa+q \nu}} .
$$

Following the argument in the proof of Theorem 3.3, a sufficient condition to have $\lim _{m \rightarrow \infty} Y_{m}=0$ is

$$
\theta \geq C S_{r}^{\frac{\nu}{\kappa}}\left(r^{-p}\|u\|_{\infty, \Psi_{0}}^{p-2}\right)^{\frac{\nu+p}{\kappa}} Y_{0}^{\frac{p}{\kappa}}
$$

so

$$
\|u\|_{\infty, \Psi} \leq C S_{r}^{\frac{\nu}{\kappa}}\left(r^{-p}\|u\|_{\infty, \Psi_{0}}^{(p-2)}\right)^{\frac{\nu+p}{\kappa}} Y_{0}^{\frac{p}{\kappa}}
$$


This proves the theorem for $q \geq 2$. To obtain the result for $1 \leq q<2$, follow the same procedure as in Theorem 3.3, with $r_{m}$ as in (3.16),

$$
\begin{aligned}
\tilde{\Psi}_{m} & =B\left(x_{0}, r-r_{m}\right) \times I_{T} \\
\tilde{\psi}_{0}(\xi, \tau) & =B\left(\xi, r_{m}\right) \times I_{T}, \\
\tilde{\psi}(\xi, \tau) & =B\left(\xi, r_{m+1}\right) \times I_{T}
\end{aligned}
$$

and replacing $r_{m}^{-p}\|u\|_{\infty, \Psi_{0}}^{p-2}+t_{m}^{-1}$ in (3.17) with $r_{m}^{-p}\|u\|_{\infty, \Psi_{0}}^{p-2}$ and $r^{-p}\|u\|_{\infty, \Psi_{0}}^{p-2}+$ $T^{-1}$ in (3.17), (3.19) and (3.20) with $r^{-p}\|u\|_{\infty, \Psi_{0}}^{p-2}$.

The mean value inequality for the heat equation (3.1) is, as said before, a special case of the two preceding theorems. To see this, let $M$ be an $n$ dimensional non-compact manifold with non-negative Ricci curvature, $n>$ 2 , and let $u$ be a solution of the heat equation in some cylinder $B\left(x_{0}, r\right) \times$ $(0, T)$. Choose $q=1$ and $p=2$ and assume $r=\sqrt{T}$ as in (3.1). Since we assume $n>2$, the local Sobolev inequality (2.12) holds with $\nu=n$, with Sobolev constant $S_{r}$ given by (2.13). In this case, both (3.15) and (3.21) coincide with (3.1).

\section{Finite propagation speed.}

In this section, we show that sub-solutions of the non-linear equation (2.1) in a non-compact manifold $M$ have finite propagation speed, using the mean value inequality from Theorem 3.4. Furthermore, we obtain a local estimate for the speed of propagation. This estimate turns out to depend on the curvature of the manifold $M$, through the curvature dependence of the constants $D_{U}$ and $P_{U}$ in (2.10) and (2.11). The key ingredient in the proof of the finite propagation speed property is an estimate for the term $r^{-p}\|u\|_{\infty, \Psi_{0}}^{p-2}$ in the mean value inequality (3.21).

The results in this section are only valid if $p>2$.

The following theorem implies Theorem 1.1 in the introduction:

Theorem 4.1. Assume that $(M, \mu)$ is a non-compact, complete, weighted Riemannian manifold. Let $T>0$, and let $u \in \mathcal{S}_{p}\left(M_{T}\right)$ be a non-negative, bounded, weak sub-solution of (2.1), where $\mathcal{A}$ satisfies (2.2) and (2.3), and suppose that there exists a ball $B\left(x_{0}, d\right)$ such that

$$
B\left(x_{0}, d\right) \cap \operatorname{supp} u(\cdot, 0)=\emptyset .
$$

For all $0 \leq t<\min \left(t_{0}, T\right)$, with

$$
t_{0}=C\left(D_{B\left(x_{0}, d\right)}^{2} P_{B\left(x_{0}, d\right)}\right)^{-\nu} d^{p}\|u(\cdot, 0)\|_{\infty, M}^{-(p-2)}, \quad C=C(p, \nu),
$$


we have $\|u(\cdot, t)\|_{\infty, B\left(x_{0}, \frac{d}{2}\right)}=0$, that is

$$
B\left(x_{0}, \frac{d}{2}\right) \cap \operatorname{supp} u(\cdot, t)=\emptyset .
$$

Proof. Since $p>2$, and $u$ is bounded, we can, for $r_{0}>0, x \in M$, define $\phi_{r_{0}, x}: I_{T} \rightarrow \mathbb{R}$ by

$$
\phi_{r_{0}, x}(t)=\sup _{0 \leq \tau<t} \sup _{r \geq r_{0}} r^{-\frac{p}{p-2}}\|u(\cdot, \tau)\|_{\infty, B(x, r)} .
$$

We will, for any $x \in B\left(x_{0}, \frac{d}{2}\right)$ and for small enough $t$, give an upper bound for $\phi_{r_{0}, x}(t)$, independent of $r_{0}$.

First, consider $r^{-\frac{p}{p-2}}\|u(\cdot, t)\|_{\infty, B(x, r)}$ for $r$ small: we assume $r<\frac{d}{4}$. In this case, for all $x \in B\left(x_{0}, \frac{d}{2}\right)$, we have $B(x, 2 r) \subset U$ and

$$
B(x, 2 r) \cap \operatorname{supp} u(\cdot, 0)=\emptyset,
$$

so for all $0 \leq t<T$, we can apply the second version of the mean value inequality, (3.21), in the cylinders $\Psi:=B(x, r) \times[0, t] \subset \Psi_{0}:=B(x, 2 r) \times$ $[0, t]$. The mean value inequality $(3.21)$ gives an estimate for $\|u(\cdot, t)\|_{\infty, \Psi}$, but since $u \in C\left(I_{T}, L^{2}(M)\right)$ it follows that, in fact, at all times $0 \leq \tau<t$

$$
\|u(\cdot, \tau)\|_{\infty, B(x, r)} \leq C S_{x, 2 r, U}^{\frac{\nu}{\kappa}}\left(r^{-p}\|u\|_{\infty, \Psi_{0}}^{p-2}\right)^{\frac{\nu+p}{\kappa}}\left(\iint_{\Psi_{0}} u^{q} d \mu d \tau\right)^{\frac{p}{\kappa}}
$$

where $U=B\left(x_{0}, d\right)$.

Taking $q=p-1$, we find that for all $0 \leq \tau<t$

$$
\begin{aligned}
& r^{-\frac{p}{p-2}}\|u(\cdot, \tau)\|_{\infty, B(x, r)} \leq C S_{x, 2 r, U}^{\frac{\nu}{\kappa}} r^{-\frac{p \nu}{\kappa}}\left(r^{-p}\|u\|_{\infty, \Psi_{0}}^{p-2}\right)^{\frac{\nu+p}{\kappa}} \\
& \times\left(\iint_{\Psi_{0}}\left(r^{-\frac{p}{p-2}} u\right)^{p-1} d \mu d \tau\right)^{\frac{p}{\kappa}} \\
& \leq C S_{x, 2 r, U}^{\frac{\nu}{\kappa}}\left(\frac{V(x, 2 r))}{r^{\nu}}\right)^{\frac{p}{\kappa}} \\
& \left(\int_{0}^{t} \phi_{r_{0}, x}^{p-1}(\tau) d \tau\right)^{\frac{p}{\kappa}} \phi_{r_{0}, x}(t)^{1-\frac{p}{\kappa}}
\end{aligned}
$$

In what follows, the value of the constant $C$ will change several times, but $C=C(p, \nu)$ everywhere. 
Using that the Sobolev constant $S_{x, 2 r, U}$ is given by (2.13) together with Young's inequality, the last estimate becomes

$$
r^{-\frac{p}{p-2}}\|u(\cdot, t)\|_{\infty, B(x, r)} \leq C\left(D^{2} P\right)^{\nu} \int_{0}^{t} \phi_{r_{0}, x}^{p-1}(\tau) d \tau+\frac{1}{2} \phi_{r_{0}, x}(t),
$$

for all $x \in B\left(x_{0}, \frac{d}{2}\right)$ and $r<\frac{d}{4}$.

Now, consider $r \geq \frac{d}{4}$, again with $x \in B\left(x_{0}, \frac{d}{2}\right)$ : since $M$ is assumed to be complete, $\mathcal{S}_{p}\left(M_{T}\right)=\stackrel{o}{\mathcal{S}}_{p}\left(M_{T}\right)$, (where $\stackrel{o}{\mathcal{S}}_{p}\left(M_{T}\right)$ is as defined in $(2.8)$ ), see for example [2, p. 34], so we can apply Lemma 2.4 to obtain that for any $t \in I_{T}$

$$
r^{-\frac{p}{p-2}}\|u(\cdot, t)\|_{\infty, B(x, r)} \leq\left(\frac{4}{d}\right)^{\frac{p}{p-2}}\|u(\cdot, 0)\|_{\infty, M} .
$$

Combining this with (4.3), we get

$$
\phi_{r_{0}, x}(t) \leq\left(2^{a} d^{-\frac{p}{p-2}}\|u(\cdot, 0)\|_{\infty, M}+C\left(D^{2} P\right)^{\nu} \int_{0}^{t} \phi_{r_{0}, x}^{p-1}(\tau) d \tau\right) .
$$

Since for all $r_{0}>0$ and for any $x \in B\left(x_{0}, \frac{d}{2}\right)$ the function $\phi_{r_{0}, x}(t)$ is bounded by $r_{0}^{-\frac{p}{p-2}}\|u(\cdot, 0)\|_{\infty, M}$, and $\phi_{r_{0}, x}(0) \leq d^{-\frac{p}{p-2}}\|u(\cdot, 0)\|_{\infty, M}, \phi_{r_{0}, x}$ is majorised by the solution to the equation

$$
f^{\prime}(t)=C\left(D^{2} P\right)^{\nu} f^{p-1}(t), \quad f(0)=2^{a} d^{-\frac{p}{p-2}}\|u(\cdot, 0)\|_{\infty, M} .
$$

Solving this equation, we obtain that for $t<\min \left(t_{0}, T\right)$, with

$$
t_{0}=C\left(D^{2} P\right)^{-\nu} d^{p}\|u(\cdot, 0)\|_{\infty, M}^{-(p-2)},
$$

(observe that $t_{0}$ is positive)

$$
\phi_{r_{0}, x}(t) \leq\left(d^{p}\|u(\cdot, 0)\|_{\infty, M}^{-(p-2)}-C\left(D^{2} P\right)^{\nu} t\right)^{-\frac{1}{p-2}}
$$

uniformly in $x \in B\left(x_{0}, \frac{d}{2}\right)$, independent of $r_{0}>0$, or

$$
\|u(\cdot, t)\|_{\infty, B(x, r)} \leq r^{\frac{p}{p-2}}\left(d^{p}\|u(\cdot, 0)\|_{\infty, M}^{-(p-2)}-C\left(D^{2} P\right)^{\nu} t\right)^{-\frac{1}{p-2}}
$$

for all $r>0$, for all $x \in B\left(x_{0}, \frac{d}{2}\right)$, implying the statement of the theorem. 
In the theorem, we require that $u$ is a weak sub-solution in $M_{T}$. This can be replaced with the requirement that $u$ is a solution in $U_{T}$ if we redefine $\phi_{r_{0}, x}($ see $(4.2))$ as

$$
\phi_{r_{0}, x}(t)=\sup _{0 \leq \tau<t r \geq r_{0}} \sup r^{-\frac{p}{p-2}}\|u(\cdot, \tau)\|_{\infty, B(x, r) \cap U},
$$

and replace all norms $\|u(\cdot, 0)\|_{\infty, M}$ with $\|u\|_{\infty, U_{T}}$.

On $\mathbb{R}^{n}$, an explicit solution of (1.1) is given by the Barenblatt solution

$$
\mathcal{B}_{p}(x, t)=t^{-\frac{n}{\kappa}}\left(c-\kappa^{\frac{1}{1-p}} \frac{p-2}{p}\left(\frac{|x|}{t^{\frac{1}{\kappa}}}\right)^{\frac{p}{p-1}}\right)_{+}^{\frac{p-1}{p-2}},
$$

where $\kappa=p+n(p-2)$. The estimate (4.1) for the time up to which the solution remains 0 is not optimal for this solution, which at $x \neq 0$ remains zero until $t_{0}=C|x|^{n(p-2)+p}$, whereas from (4.1), we get $t_{0}=C|x|^{p}$.

We conclude this paper with estimates for the growth of the support of a weak solution of the non-linear equation (2.1) in a non-compact manifold. The following theorem implies Theorem 1.2.

Theorem 4.2. Let $(M, \mu)$ be a non-compact, complete, weighted Riemannian manifold, and let $u \in \mathcal{S}_{p}\left(M \times \mathbb{R}^{+}\right)$be a non-negative, bounded, weak sub-solution of (2.1), where $\mathcal{A}$ is assumed to satisfy (2.2) and (2.3). Assume that $\operatorname{supp} u(\cdot, 0)$ is compact. For $r>0$, define the $r$-neighbourhood of $\operatorname{supp} u(\cdot, 0)$,

$$
U_{r}=\{x \in M: \operatorname{dist}(x, \operatorname{supp} u(\cdot, 0)) \leq r\},
$$

and let

$$
T=\sup _{r>0} C\left(D_{U_{3 r}}^{2} P_{U_{3 r}}\right)^{-\nu} r^{p}\|u(\cdot, 0)\|_{\infty, M}^{-(p-2)}
$$

where $C=C(p, \nu)$ is the constant from (4.1).

There exists an increasing, non-negative function

$$
r:[0, T) \rightarrow \mathbb{R}
$$

such that for any $0<t<T$,

$$
\operatorname{supp} u(\cdot, t) \subset U_{r(t)} \text {. }
$$


Proof. Let $r>0$ and define

$$
A_{r}=\{x \in M: r<\operatorname{dist}(x, \operatorname{supp} u(\cdot, 0))<2 r\} .
$$

Choose a finite number of points $x_{i} \in A_{r}$ such that

$$
A_{r} \subset \cup_{i} B\left(x_{i}, \frac{r}{2}\right) .
$$

For any of the $x_{i}, B\left(x_{i}, r\right) \cap \operatorname{supp} u(\cdot, 0)=\emptyset$, so by Theorem 4.1

$$
B\left(x_{i}, \frac{r}{2}\right) \cap \operatorname{supp} u(\cdot, t)=\emptyset
$$

for all

$$
t<t_{\left(x_{i}, r\right)}=C\left(D_{B\left(x_{i}, r\right)}^{2} P_{B\left(x_{i}, r\right)}\right)^{-\nu} r^{p}\|u(\cdot, 0)\|_{\infty, M}^{-(p-2)},
$$

where $C$ is the constant from (4.1). Since $B\left(x_{i}, r\right) \subset U_{3 r}$ for all $x_{i}, D_{B\left(x_{i}, r\right)} \leq$ $D_{U_{3 r}}, P_{B\left(x_{i}, r\right)} \leq P_{U_{3 r}}$, and

$$
t_{\left(x_{i}, r\right)} \geq t(r)=C\left(D_{U_{3 r}}^{2} P_{U_{3 r}}\right)^{-\nu} r^{p}\|u(\cdot, 0)\|_{\infty, M}^{-(p-2)}
$$

for all $x_{i}$. By (4.8), this implies that for all $t<t(r)$

$$
A_{r} \cap \operatorname{supp} u(\cdot, t)=\emptyset \text {. }
$$

In fact, for $t<t(r)$,

$$
\operatorname{supp} u(\cdot, t) \subset U_{r}:
$$

given $1>\varepsilon>0$ and $\rho>0$, let $\eta$ be a smooth, non-negative function on $M \backslash U_{r}$ such that $0 \leq \eta \leq 1, \eta \equiv 1$ on

$$
V_{\rho}=\left\{x \in M \backslash U_{r}: \operatorname{dist}\left(x, U_{r}\right)<\rho\right\},
$$

$\eta \equiv 0$ on $M \backslash V_{\frac{2 \rho}{\varepsilon}}$ and $|\nabla \eta|<\frac{\varepsilon}{\rho}$. Observe that for $h>0,0 \leq t<t(r)-h$, $u_{h} \eta^{p} \in L^{2}\left(M \backslash U_{r}\right) \cap \stackrel{o}{W^{1, p}}\left(M \backslash U_{r}\right)$, with $u_{h}$ the Steklov average of $u$, so proceeding as in the proof of Lemma 3.1 (with $t_{1}=0$ ), we find that for any $t<t(r)$

$$
\int_{V_{\rho}} u^{2}(\cdot, t) d \mu \leq \varepsilon^{p} C\|u(\cdot, 0)\|_{\infty, M}^{p-2} \int_{0}^{t} \int_{M} u^{2} d \mu d t .
$$

Since $\varepsilon$ and $\rho$ are arbitrary, this shows that $\operatorname{supp} u(\cdot, t) \subset U_{r}$ for all $t<t(r)$.

If $t<T$, by Definition (4.6), there exists $r>0$ such that $t<t(r)$, and hence $\operatorname{supp} u(\cdot, t) \subset U_{r}$. Define

$$
r(t)=\inf \{r: t<t(r)\} .
$$


Then $\operatorname{supp} u(\cdot, t) \subset U_{r(t)}$, and the function $r(t)$ satisfies the conditions in the theorem.

If

$$
T=\sup _{r>0} C\left(D_{U_{3 r}}^{2} P_{U_{3 r}}\right)^{-\nu} r^{p}\|u(\cdot, 0)\|_{\infty, M}^{-(p-2)}
$$

as defined in (4.6) is finite, these estimates are only local in time. However, under certain conditions on the Ricci curvature of $M$, global estimates for the speed of propagation of non-negative solutions of (2.1) can be given:

Corollary 4.3. Let $M$ be a non-compact, complete Riemannian manifold with metric $g$ and let $u \in \mathcal{S}_{p}\left(M \times \mathbb{R}^{+}\right)$be a non-negative, bounded, weak sub-solution of (2.1), where $\mathcal{A}$ is assumed to satisfy (2.2) and (2.3).

If $\operatorname{supp} u(\cdot, 0)$ is compact, and the Ricci curvature of $M$ satisfies for all $x \notin \operatorname{supp} u(\cdot, 0)$

$$
\operatorname{Ric}_{M}(x) \geq-\frac{c(n-1)}{\operatorname{dist}(x, \operatorname{supp} u(\cdot, 0))^{2}} g
$$

then for all $t>0$,

$$
\operatorname{supp} u(\cdot, t) \subset U_{r(t)},
$$

where $U_{r(t)}$ was defined in (4.5) and

$$
r(t)=C\left(\|u(\cdot, 0)\|_{\infty, M}^{p-2} t\right)^{\frac{1}{p}}
$$

Proof. The proof is similar to that of Theorem 4.2. For $r>0$, define $A_{r}$ as in (4.7), and choose a finite number of points $x_{i} \in A_{r}$ such that

$$
A_{r} \subset \cup_{i} B\left(x_{i}, \frac{r}{4}\right) .
$$

By Theorem 4.1,

$$
B\left(x_{i}, \frac{r}{4}\right) \cap \operatorname{supp} u(\cdot, t)=\emptyset
$$

for all

$$
t<t_{\left(x_{i}, r\right)}=C\left(D_{B\left(x_{i}, \frac{r}{2}\right)}^{2} P_{B\left(x_{i}, \frac{r}{2}\right)}\right)^{-\nu} r^{p}\|u(\cdot, 0)\|_{\infty, M}^{-(p-2)} .
$$

On each of the $B\left(x_{i}, \frac{r}{2}\right)$, the Ricci curvature is bounded from below by

$$
-\frac{4 c(n-1)}{r^{2}} g
$$


so by (2.14), there exists $C>0$, independent of $x_{i}$ and $r$, such that

$$
D_{B\left(x_{i}, \frac{r}{2}\right)}, P_{B\left(x_{i}, \frac{r}{2}\right)} \leq C
$$

This implies

$$
t\left(x_{i}, r\right) \geq \tau(r)=C r^{p}\|u(\cdot, 0)\|_{\infty, M}^{-(p-2)},
$$

where again the constant $C$ is independent of $x_{i}$ and $r$. Proceeding as in the proof of Theorem 4.2, it can be shown that for all $t<\tau(r), \operatorname{supp} u(\cdot, t) \subset U_{r}$, and hence, if we define

$$
r(t)=C\left(\|u(\cdot, t)\|_{\infty, M}^{p-2} t\right)^{\frac{1}{p}}
$$

then

$$
\operatorname{supp} u(\cdot, t) \subset U_{r(t)}
$$

In Corollary 4.3, the manifold $M$ is assumed to be an unweighted manifold. However, if $M$ is weighted with weight $\sigma$ and there exist constants $0<c, C$ such that $c<\sigma<C$, then the corollary continues to hold in $M$, since it follows immediately from the definition of the constants $D_{U}$ and $P_{U}$ in (2.10) and (2.11) that the estimate (4.9), from which the corollary follows, remains valid.

\section{Acknowledgement.}

The author would like to thank Alexander Grigor'yan for many helpful and stimulating discussions.

\section{References.}

[1] C. Atkinson and J.E. Bouillet. Some qualitative properties of solutions of a generalised diffusion equation. Math. Proc. Cambridge Philos. Soc., 86:495-510, 1979.

[2] Th. Aubin. Nonlinear analysis on manifolds. Monge-Ampére Equations, volume 252 of Grundlehren der mathematischen Wissenschaften. Springer-Verlag New York, 1982.

[3] G.I. Barenblatt. On self-similar motions of compressible uids in a porous medium. Prikl. Mat. Mekh., 16:679-698, 1952. in Russian. 
[4] J.E. Bouillet and C. Atkinson. A generalized diffusion equation: Radial symmetries and comparison theorems. J. Math. Anal. Appl., 95(1):37$68,1983$.

[5] P. Buser. A note on the isoperimetric constant. Ann. Sci. Ecole Norm. Sup., 15:213-230, 1982.

[6] S.A.J. Dekkers. Degenerate parabolic equations on Riemannian manifolds. PhD thesis, Imperial College, London, 2003.

[7] J.I. Diaz and M.A. Herrero. Estimates on the support of the solutions of some nonlinear elliptic and parabolic problems. Proc. Roy. Soc. Edinburgh, 89A:249-258, 1981.

[8] E. DiBenedetto. Degenerate Parabolic Equations. Springer-Verlag, 1993.

[9] E. DiBenedetto and M.A. Herrero. On the Cauchy problem and initial traces for a degenerate parabolic equation. Trans. Amer. Math. Soc., 314(1):187-224, 1989.

[10] A. Grigor'yan. The heat equation on noncompact Riemannian manifolds. Math. USSR-Sb., 72:47-77, 1992.

[11] A. Grigor'yan. Estimates of heat kernels on Riemannian manifolds. In E.B. Davies and Yu. Safarov, editors, Spectral Theory and Geometry. ICMS Instructional Conference, Edinburgh 1998, volume 273 of London Math. Soc. Lecture Notes Series, pages 140-225. Cambridge Univ. Press, 1999.

[12] M. Gromov. Metric Structures for Riemannian and Non-Riemannian Spaces, volume 152 of Progress in Mathematics. Birkhäuser, 1998.

[13] A.S. Kalashnikov. Some problems of the qualitative theory of non-linear degenerate second-order parabolic equations. Russian Math. Surveys, 42(2):169-222, 1987.

[14] S. Kamin and J.L. Vázques. Fundamental solutions and asymptotic behaviour for the $p$-Laplacian equation. Rev. Mat. Iberoamericana, $4(2): 339-354,1988$.

[15] T. Kilpeläinen and P. Lindqvist. On the Dirichlet boundary value problem for a degenerate parabolic equation. SIAM J. Math. Anal., 27(3):661-683, 1996. 
[16] O.A. Ladyzenskaja, V.A. Solonnikov, and N.N. Uralceva. Linear and Quasilinear Equations of Parabolic Type, volume 23 of Translations of Mathematical Monographs. American Mathematical Society, 1968.

[17] P. Li and R. Schoen. $L^{p}$ and mean value properties of subharmonic functions on Riemannian manifolds. Acta Math., 153:279-301, 1984.

[18] R.E. Pattle. Diffusion from an instantaneous point source with a concentration-dependent coefficient. Quart. J. Mech. Appl. Math., 12:407-409, 1959 .

[19] L. Saloff-Coste. A note on Poincaré, Sobolev and Harnack inequalities. Internat. Math. Res. Notices, 2:27-38, 1992.

[20] L. Saloff-Coste. Aspects of Sobolev-Type Inequalities, volume 289 of London Mathematical Society Lecture notes series. Cambridge University Press, 2001.

Department of Mathematics

UTRECHT UNIVERSITY

The Netherlands

E-mail address: dekkers@math.uu.nl

RECeived July 2, 2004. 